particular value to the industrial chemist anxious to keep "right up to date on the literature".

The course of such an experimental system will be watched with great interest. Its eventual success may well depend on the cost of its production and it remains to be seen whether American machines directed by Malcolm Dysón can do a cheaper and more useful job for chemical documentation than is being done at present by the carefully selective man- and woman. power behind Current Chemical Papers. M. STACEY

\title{
MEASUREMENT AND CONTROL OF MOIRÉ FRINGES
}

$\mathrm{T}$ HE illustrated booklet "Moiré Fringes for Measurement and Control", prepared by the National Physical Laboratory and the National Engineering Laboratory for members of the machine-tool and engineering industries, gives a brie $s$ account of the essential features of moiré-fringe methods and some of their engineering applications (Pp. 11. London : Department of Scientific and Industrial Research, 1960).

Moiré fringes are the pattern of relatively broad equidistant bands produced when two gratings with equal-line spacings are placed face to face, but with the sets of lines not quite parallel to each other. When one of the gratings is moved in the direction at right angles to the lines on it, the fringes move through exactly one fringe spacing as the fringe moves through one grating-line spacing. The variation in brightness as the fringe moves can be detected by a photoelectric cell and the linear movement can therefore be converted into a corresponding electrical signal. Gratings can be produced with radial lines also and thus angular movement can be measured or recorded electrically. Methods for the production of gratings at a reasonable price in the quantities and sizes for industrial use have recently been developed, and copies of master gratings produced at the National Physical Laboratory and National Engineering Laboratory are now available commercially. For most engineering applications gratings with about 1,000 lines per inch are required, but gratings up to
10 in. long with up to 10,000 lines per inch or even glass gratings up to $3 \mathrm{ft}$. long for special purposes can be produced.

Since moiré fringes are produced by very many accurately spaced grating lines and the effect of any errors of spacing of individual lines is reduced by the averaging process which occurs in the production of fringes, great accuracy in measurement is obtainable by using the fringes. Both analogue and digital systems can be used to handle the photoelectric signals, and various applications related to the automatic positioning of machine tools are described in the booklet. It is emphasized that the successful use of moiré-fringe systems requires specialized optical and electronic techniques and that to obtain maximum advantage from the use of moiré fringes in the machine tool field, close co-operation is required between the mechanical engineer and optical and electronic experts. Future developments of the application of the moiré-fringe technique may revolutionize the design of machine tools and the accuracy of their products.

The secondment of members of the staffs of industrial firms to the National Physical Laboratory and National Engineering Laboratory would be a particularly valuable way of speeding up progress, and the laboratories extend, in the booklet, an invitation to firms with electronic, optical or engineering interests to work with them on projects of mutual interest.

\section{GRAFT VERSUS HOST REACTIONS ON THE CHORIOALLANTOIC MEMBRANE OF THE CHICK EMBRYO}

\author{
By SIR MACFARLANE BURNET, O.M., F.R.S., and DEBORAH BURNET \\ Walter and Eliza Hall Institute of Medical Research, Melbourne, Australia
}

\begin{abstract}
$\mathrm{T}$ HE clonal selection theory of immunity ${ }^{1}$ in its present form postulates that in the normal animal a large proportion (perhaps all) of the mesenchymal cells could be divided into clones, each of which is pre-adapted to react with one or other of two antigenic determinant patterns. (The alternatives of being able to react with one, three or four such determinants have not yet been excluded.) The initial distribution of these reactivities is essentially at random, and in the present context there is no call to discuss how the patterns have arisen or how this random distribution to the ancestors of the marked clones could be accomplished. By hypothesis, in the adult there are no clones which can react with normal accessible components of the individual concermed, but there are present clones representing
\end{abstract}

all types of antigenic determinant not present in the body.

Antibody production is presumed to occur in two phases corresponding (1) to the inductive phase and (2) to the productive phase of orthodox immunological theory. In the inductive phase two processes occur : there is proliferation of the clone to produce many more members and the newly produced cells have an increased reactivity with the corresponding antigenic determinant. In the second phase, renewed contact with the antigenic determinant results in proliferation, conversion to the plasma cell form and production of antibody.

According to orthodox immunological theory (for example, ref. 2), the interpretation of the secondary productive phase is not significantly different 